\title{
SOUTH PARK ET LA MORALE
}

\begin{abstract}
Aurore Courte ${ }^{2}$
La série animée South Park se caractérise dès les premiers regards par le caractère rudimentaire de son animation, la vulgarité de ses dialogues et la violence qu'elle met en scène. Durant une vingtaine de minutes, humour scatologique, situations sexuellement embarrassantes et grossièretés en tout genre se mêlent au détournement de l'antisémitisme, autant que des stars, des institutions ou des débats de société. Rien ne semble pouvoir être épargné par ce rouleau compresseur de l'irrévérence. Pourtant, invariablement, un quatrième élément vient compléter la recette de cette production médiatique : une morale.

Aussi, la fiction la plus encline à flirter avec l'immoralisme se trouve-t-elle également être celle qui exprime l'univers moral dans lequel elle s'insère, et dont elle est en même temps l'expression, de la manière la plus explicite. Telles les deux faces d'une même pièce, moralisme et immoralisme s'entremêlent ici, comme pour nous rappeler
\end{abstract}

1 Créée par Trey Parker et Matt Stone, South Park est une série animée diffusée par Comedy Central depuis le 13 août 1997. Elle fait son apparition en France un an plus tard sur Canal Plus, bientôt suivi par Club RTL, Comédie et Plug TV.

2 Cet article est basé sur le mémoire de Master en information et communication présenté à l'UCL en 2006.

Recherches en communication, $\mathrm{n}^{\circ} 25$ (2006). 
que ces positions n'en forment en fait qu'une seule et renferment l'ensemble des possibles, rejetant l'amoralisme parmi les idées sans lieu.

Parler d'une morale de South Park et prétendre l'analyser ne semble donc pas dépourvu de fondement. Mais qu'entend-on par morale?

La morale, telle que nous l'entendrons pour l'élaboration de cette analyse en tout cas, a partie liée avec trois points d'ancrage : le sujet, la raison et la société. Le sujet, tout d'abord, se présente comme le fondement de l'action libre. C'est lui qui sera porteur des questions telles que la volonté, le libre arbitre et la responsabilité. Sans lui, un système moral n'a pas de raison d'être. La raison, ensuite, correspond à cette partie de l'être humain qui lui permet de se détacher de son existence particulière pour se rapprocher de l'universel. C'est grâce à elle que nous élaborons des normes devant permettre l'exercice rationnel de l'action. Elle nous permet de construire un système de droits et de devoirs, ainsi que d'envisager des horizons à l'agir moral tels que l'égalité, la justice... La société, enfin, constitue la base de coutumes, de croyances et de pratiques sur laquelle se construit toute morale. Le but de nombreux philosophes a souvent été de s'en défaire grâce à l'exercice de la raison afin de donner un véritable fondement à l'action humaine. Cette entreprise est perdue d'avance et la plupart n'ont réussi qu'à reconstruire la morale de leur temps en l'affublant d'apparats sensés la rendre universelle. Au lieu de s'acharner dans cette voie, nous admettons donc la société comme un des trois piliers de l'action humaine déterminant la frontière primordiale entre le bien et le mal.

South Park se situe au centre de ces questions, tant par les positions qu'il défend explicitement, généralement à la fin de l'épisode, que par l'univers de préoccupations dans lequel il s'insère. Il ne faudrait cependant pas en conclure trop vite que la série nous offre un système moral rationnel, clef en main, qu'il ne nous resterait plus qu'à déconstruire afin d'en découvrir les fondements, les idées maîtresses, les tenants et aboutissants. Une production médiatique est une entité vivante et polymorphe. Bien sûr, elle a été créée et existe en tant qu'objet délimité, mais à bien des égards elle se révèle beaucoup plus proche d'une pratique sociale, avec ses règles de fonctionnement propres.

South Park n'est pas un système. Il s'agit avant tout d'une série télévisée. En tant que telle, elle suppose une intégration au sein d'une entité plus vaste et protéiforme encore que serait la culture médiatique. Elle s'intègre également à des pratiques, voire des rituels, de réception et de consommation. Elle inscrit sa présence au cœur de l'intimité et du quotidien des familles. Elle répond à des règles de construction du récit 
particulières. $\mathrm{Si}$, au cours de cet article, nous tenterons donc d'inscrire les différents éléments donnant une teneur morale à la série dans un cadre logique et cohérent, nous devrons néanmoins garder à l'esprit le caractère artificiel et figé d'une telle entreprise par rapport à l'événement télévisé en lui-même.

\section{Le langage}

C'est donc le genre "feuilleton" qui fait de Dallas, comme de tous les autres feuilletons, un "dire de l'être". On pourrait dire la même chose des séries télévisées. Tout simplement parce qu'un réalisateur espère vendre le plus grand nombre possible d'épisodes, il fabriquera un feuilleton ou une série, un langage et non un texte, il s'inscrira donc dans un temps circulaire, usera d'une diction formulaire ${ }^{1}$.

L'analyse que fait Florence Dupont de Dallas reste d'une pertinence fulgurante lorsqu'il s'agit d'appliquer la formule à South Park. Ainsi, dans la structuration du récit, un premier point saute directement aux yeux, c'est la rupture radicale qui y est opérée entre les enfants et les adultes. La série semble présenter une vision duelle du monde dans lequel la naïveté, autant que l'humour, le désengagement et le pragmatisme seraient l'apanage des petits tandis que l'idéologie, l'aveuglement et l'absence d'humour seraient celui des grands.

De leur côté, les personnages, eux aussi, portent largement l'univers symbolique de la série. Alors que leur construction graphique et psychologique est réduite à sa plus simple expression, leur fixation en nature est clairement revendiquée. Rien ne vient en nuancer le portrait mais tout concourt au contraire à en conforter l'image. Ils ne peuvent ni changer, ni évoluer, ni même trouver d'explications hors d'eux-mêmes. Ainsi, Eric Cartman pourra être envoyé en camps de régime, maté par un dresseur de chien ou devenir le grand prêtre de sa propre religion; rien ne pourra le transformer ni physiquement, ni moralement.

Mais plus que tout, South Park nous dit South Park. Il nous dévoile son langage; il nous invite à apprécier sa nouvelle mise en forme. Rien n'est caché, ni surprenant et c'est cette reconnaissance du même toujours renouvelé qui provoque le plaisir. On intègre ainsi une

1 DuPONT, F., Homère et Dallas, Paris, Hachette, 1991, p. 123. 
communauté de langage. Regarder la série, ce n'est pas découvrir une histoire, c'est avant tout réaffirmer son appartenance à la culture que celle-ci met en scène.

Cette fonction de distinction repose cependant sur un fondement commun dans toutes les sociétés démocratiques, à savoir la liberté d'expression. Si South Park peut se permettre ce qu'il fait et qui est à la base d'une certaine distinction, c'est en effet grâce à ce droit fondamental. Ce qui est célébré, au delà de l'appartenance à une communauté, c'est la liberté dont jouit chacun de former des langages qui lui sont propres et qui lui permettront de fonder des complicités en dehors, mais grâce à un pouvoir central. Le thème de la célébration que renouvelle la série à chaque épisode pourrait donc être celui-ci : qu'il est bon d'être jeune et irrévérencieux dans une Amérique libre.

Ainsi, la série n'est en rien une description, même partielle, de la société américaine. Il s'agit avant tout d'une pratique, de la célébration d'une culture médiatique de la libre expression, de l'ironie et de l'impertinence. Cette culture implique une certaine conception de la nature humaine : à la fois cynique, perdue, et en recherche, capable par ses seules aptitudes d'adopter la voie du bon sens. S'il est un fondement moral qui se trouve valorisé ici, c'est donc bien celui qu'apporte la conviction en un individu libre et responsable de ses actes et de son être au-delà de toutes les excuses que l'on pourrait lui trouver par ailleurs. L'individu se présente alors comme la seule base possible à tout raisonnement moral, puisque lui seul pourra en être tenu pour responsable. Cette construction individuelle ne se trouve pas transfiguré par une entité supérieure, telle que la raison ou la société, mais elle reste au niveau d'un bricolage personnel entre pratique et réflexion, entre société et raison : une morale du bon sens.

La culture induite, autant que construite, par cette série est donc double. Tout d'abord, ce dessin animé se propose de donner un point de vue moyen, ni idéologique, ni utilitariste, sur les problèmes moraux qu'il aborde. Une telle position suppose déjà de fameux à priori, tels que la capacité de tout le monde à atteindre une position morale raisonnée, la garantie qu'apporte le désengagement par rapport à l'aveuglement idéologique pour atteindre celle-ci et une certaine idéalisation du mode de pensée enfantin. Ensuite, il s'agit d'une culture de la distinction entre les jeunes et les autres. En effet, ce qui est célébré avant tout, c'est la vivacité de l'esprit de subversion par rapport aux valeurs figées. 


\section{Les ressorts}

Certains traits du dessin animé que nous analysons ici s'imposent immédiatement au téléspectateur. Cela s'explique facilement par leur caractère inhabituel. Ainsi, représentation crue du corps, humour scatologique et transgression de tabous sont autant de lieux communs de la diction formulaire de South Park qui lui permet de se distinguer des autres. Ces topiques, s'ils ont pour effets recherchés de choquer, n'en soutiennent pas moins tout un système de significations, autant que de fonctions, dans lequel tout se recoupe en un vaste réseau.

Nous avons choisi, pour illustrer notre propos, trois d'entre eux, qui sont apparus comme principaux. Il s'agit de la violence, de l'humour et de la subversion. Autour de chacune de ces notions se joue une lutte sans cesse à renouveler, et c'est elle qui va permettre au système de prendre vie. Ainsi, la violence ne peut être prise en compte que si elle se trouve mise en présence de l'innocence ; l'humour, que s'il est contrebalancé par la morale du bon sens commun ; enfin, la subversion que si elle se voit confrontée à la perpétuation du même.

\section{Violence et innocence}

South Park peut être estimé violent à plusieurs niveaux : le caractère cru des dialogues ; la représentation « gore » du corps, tant dans son aspect sexuel que mortel ; l'extrémisme de certaines idées exprimées, que ce soit la misogynie de monsieur Garrison ou l'antisémitisme de Cartman, par exemple ; le caractère disproportionné des conséquences entrainées par chaque acte; enfin, la violence symbolique que peut revêtir la représentation détournée de certains piliers de la culture occidentale, tels que Dieu en petit animal informe, le président Kennedy et Lady Diana aux côtés d'Hitler et de Mao en enfer ou même l'introduction d'un gentil Charles Manson dans un épisode de Noël.

Tous ces aspects participent à l'impression générale d'être face à un dessin animé violent, dont l'accès est d'ailleurs interdit aux moins de dix ans chez nous, mais aux moins de dix-huit ans aux Etats-Unis. Ce serait cependant passer à côté de l'essentiel que de n'y voir que cela. En effet, l'expression de cette violence n'est permise que par la présence d'un second élément, à savoir l'innocence. L'innocence est partout présente dans le dessin animé, même si son caractère moins agressif la rend moins visible. Ainsi, du choix de l'animation comme mode d'expression privilégié à la représentation de héros de huit ans, du carac- 
tère ludique dans la construction de la fiction à l'expression d'une voix naïve, pure de toute idéologie, révélant le chemin à suivre dans tout son bon sens, South Park nous dit l'innocence de son monde.

La conséquence de cette mise en présence de la violence et de l'innocence ne se limite cependant pas à une annulation pure et simple de l'une par l'autre. Au contraire, chaque élément garde son entière signification dans le jeu qui s'instaure entre les deux. Ainsi, l'innocence n'est pleinement innocente que dans le rapport qu'elle entretient avec la violence et celle-ci n'est jamais accomplie de manière aussi parfaite que lorsqu'elle se voit soutenue par celle-là.

Ce dont le meurtrier réel ne se souvient pas et qui lui fait évoquer l'animal en lui, n'est-ce pas l'innocence d'un désir "naturel", primitif, d'une sensualité fusionnelle, agressive et sans parole'.

Les pires choses peuvent se produire, les héros de la série gardent toujours leur fraîcheur d'enfants ; mais, dans le même temps, l'expression gratuite de la violence, là où celle-ci peut se déployer pleinement en tant que telle, a lieu lorsqu'elle est assumée par ces mêmes enfants. Les adultes font également preuve d'une extrême sauvagerie mais il s'agit alors toujours de la conséquence d'un engagement idéologique excessif.

Cet amalgame entre innocence et violence correspond à une conception culturellement marquée, comme nous le rappelle Pascal Bruckner dans son livre, La tentation de l'innocence. Selon lui, c'est de Rousseau que date l'alliance entre l'enfant et le sauvage. Ce rapprochement implique une image idéalisée d'un être vivant en communion immédiate avec les choses, donc plus proche de leur vérité intime, non encore altéré par la civilisation. Or, n'est-ce pas exactement ce type de représentation que nous avons mis en lumière dans la première partie à travers la vision duelle du monde proposée entre les enfants et les adultes, les premiers assumant une position pragmatique et désengagée tandis que les seconds servent de support à toutes sortes d'idéologies menant irrémédiablement à l'aveuglement destructeur.

L'enfant est désormais notre "bon sauvage à domicile" (Peter Sloterdijk), celui qui délivre des paroles essentielles et nous

1 DUClOS, D., Le complexe du loup garou: la fascination de la violence dans la culture américaine, Paris, La Découverte, 1994, p. 157. 
conduit vers les rives enchanteresses de la candeur. En dépit ou plutôt à cause de sa faiblesse, il sait tout mieux que nous, il est habilité presque à devenir le parent de ses parents. A notre époque où "la pédagogie est devenue théologie", nous lui confions le soin d'instruire l'adulte, nous conférons à sa puérilité une valeur telle qu'elle se renverse en supériorité ${ }^{1}$.

Ces mots, offrant un diagnostic sur notre société actuelle, trouvent un véritable écho dans les épisodes de South Park. L'enfant y est à la fois le sauvage et l'éducateur, celui qui remet de l'ordre, énonce la morale, permet le retour à l'équilibre. Bien plus qu'un simple jeu, cette dialectique instaurée par le dessin animé entre innocence et violence révèle une lame de fond de notre vision du monde. Les deux faces de cette représentation se trouvent ainsi affirmées : la joie de s'abandonner à l'égoïsme candide mais impérieux de l'enfance et la supériorité de ce point de vue naif dans le domaine de la morale.

Cependant, alors que cette image de l'enfance se retrouve de manière idéalisée dans de nombreuses productions culturelles contemporaines, comme chez Disney², ce dessin animé ne se contente pas de faire de la violence une simple prémisse théorique à la célébration du caractère de nature de la morale de l'innocence. Au contraire, elle y est omniprésente, maintenue en perpétuelle tension avec celle-ci. Aussi, même si ce sont bel et bien les enfants qui assument le rôle d'énonciateur d'une parole vraie car capable de résoudre les conflits, ils se retrouvent la plupart du temps investis de cette mission contre leur gré. La représentation de la valorisation de l'innocence se trouve donc ainsi en permettre la critique. En effet, tout en enfonçant le clou de la description des adultes comme incapables de régler leurs problèmes raisonnablement par eux-mêmes, la série nous donne aussi à voir l'absurdité de la tentation, toute aussi adulte par ailleurs, de confier aux enfants le soin de le faire à leur place. Nous nous trouvons donc ici face à une ambiguïté manifeste, puisque le fait que les enfants ne sont en rien plus proches d'une vérité essentielle se trouve être une vérité connue des intéressés seuls. En ce sens, nous pouvons comprendre l'importance du caractère vivant d'un tel univers symbolique. En effet, la tension ne peut être résolue. Le dialogue entre ces deux parts de vérité doit rester

1 BRUCKNER, P., La tentation de l'innocence, Paris, Grasset, 1995, p. 95.

2 Ibid, p. 113. 
permanent car c'est lui qui permet la vitalité et la richesse du système. C'est aussi lui qui en interdit toute fixation en une structure rigide.

Si la violence sert de complément antinomique et en même temps essentiel à l'innocence, nous pouvons également considérer qu'elle a une fonction en tant que telle. Il s'agit, en effet, d'un élément extrêmement visible et revendiqué et on ne peut se contenter d'y voir le pendant négatif de l'innocence, ne serait-ce que parce qu'il est partout aussi présent, même dans le chef des adultes. Or, Denis Duclos nous offre une raison d'être particulièrement intéressante à cette représentation de la brutalité.

L'adhésion des américains à cette culture de l'hésitation et de l'équilibre entre les principes de violence et de loi ne découle pas seulement de leur idéal de société. (...) Il y a aussi une double érotisation : celle de la "part maudite", notre réserve de spontanéité sauvage, et celle de la pudeur victorienne et de son ordre intangible, qui donne du prix aux forces interdites qu'il refoule. Sans le côté civil, bien pensant, clean, innocent, le fond meurtrier, sauvage et implacable ne saurait se conserver, mais inversement, sans l'énergie obscure de celui-ci, on semble croire que la civilisation n'aurait pas de raison d'être, en tant que rempart à la barbarie ${ }^{1}$.

L'équilibre bien-mal, retrouvé dans toutes les fictions ou le commentaire des crimes, n'est pas seulement une idée parmi d'autres, un peu bébête ou infantile, mais le fondement même des valeurs anglo-américaines cherchant à associer liberté et ordre, énergie et contrôle social en un face à face aussi prolongé que possible ${ }^{2}$.

La représentation de la violence aurait donc également pour fonction de rappeler la nécessité de l'ordre social. Loin de détruire le sens des valeurs par le caractère brutal de sa représentation, la violence en permet la justification. En ce sens, nous pouvons dire que celleci constitue le fondement sur lequel repose la prétention de la série à élaborer une position morale.

1 DuClOS, D., Op. cit., p. 245.

2 Ibid., p. 243. 


\section{Humour et morale}

L'humour revêt cette particularité de permettre à la fois la distanciation par rapport aux normes de la vie commune et de rappeler le sentiment d'appartenance à cette même communauté.

L'humour est une forme d' anthropologie sociale critique, rendant déroutant le familier, démythifiant l'exotique et retournant le monde du sens commun ${ }^{1}$.

L'humour est toujours local et particulier. Il nous introduit à une communauté (rapidité de l'intimité établie) tout en nous conférant le sentiment palpable de notre exception culturelle, voire de notre supériorité. Il nous protège de l'étrangeté, l'inconnu. Il nous relie à un ethos et à un ethnos ${ }^{2}$.

Son lien avec la morale, en tant que construction sociale fondée sur une certaine pratique quotidienne et un univers de références communes, est donc particulièrement étroit. Il peut cependant se réaliser de deux manières différentes : soit par un rire franc, raisonnable et moralisant, dénonçant le ridicule et les écarts avec la norme ; soit par un rire subversif, joyeux, dionysiaque, remettant en question le sacré et lui permettant ainsi de renaître.

Face à cette palette de l'humour, s'étendant entre celui qui resserre les liens de la communauté et celui qui les remet en cause, quelle est la conception de la morale qui se trouve mise en oeuvre ? Une chose est certaine, c'est qu'il s'agira de toute façon d'une morale inscrite dans la pratique quotidienne d'une communauté. En effet, l'humour, quels que soient les aspects qu'il revêt, a trait à ce sentiment d'appartenance tiré à des habitudes et des connaissances communes. Il ne peut prétendre fonder en logique et en raison les normes qui lui permettent de se déployer. Par contre, il jouera sur l'ambiguité entre culture et nature qu'entretient toute société. Le fondement social de la morale se trouve donc mis en valeur par l'exercice humoristique. Mais dans le même temps, la fonction subversive de l'humour exige un second fondement : l'individu, seule entité capable de liberté vis-à-vis des normes de la communauté dont il fait partie.

1 CRITCHLEY, S., De l'humour, (trad. N. PINET), Paris, Kimé, 2004, p. 67.

2 Ibid., pp. 69-70. 
Une telle morale, fondée non pas en raison mais en pratique, par un individu à la fois libre et intégré à une culture particulière, c'est ce que nous appellerons une morale pragmatique du bon sens commun. Nous étudierons plus loin en quoi une telle dénomination peut se confirmer, ou pas, par un rapprochement avec les définitions philosophiques correspondantes. En attendant, nous la maintiendrons comme telle car elle permet la prise en compte de ses trois éléments fondateurs : l'ancrage pratique, le partage par une communauté et le fondement en vérité, assumé non par un raisonnement logique bien construit mais par les résultats pragmatiques en découlant.

Rappelons pour être bien clair que cette spécification de la morale mise en œuvre par l'usage de l'humour ne porte pas ici sur les contenus moraux énoncés comme tels à la fin de la plupart des épisodes du dessin animé. Il s'agit de repérer les formes de morale sous-tendues par l'usage systématique de l'humour dans le fonctionnement propre de la série. Cependant, on ne peut prétendre traiter séparément le message et le contexte de son énonciation. Or, si les morales énoncées au sein de la plupart des épisodes ont une portée propre, on ne peut nier que l'ambiance de dérision généralisée dans laquelle elles apparaissent en atténue le poids. En effet, si l'humour en tant que tel charrie un passif important par rapport aux normes sociales, il s'agit toujours d'évidences qu'il n'est pas nécessaire d'expliciter. Dans le cas des positions énoncées par un personnage particulier au sein du récit, dont l'objet principal est quand même de faire rire, leur contenu n'aura qu'une importance secondaire. La dérision en atténue la portée autant qu'elle en permet l'instauration par son travail de sape. Ainsi, il est vrai que la prétention à la vérité en sera augmentée par le fait qu'elles s'édifient sur les ruines encore fumantes des idéologies désacralisées, mais si l'on n'y adhère pas, l'épisode n'en est pas pour autant discrédité car il aura tenu sa promesse principale, celle de nous divertir.

Le lien entre humour et morale se situe donc sur deux tableaux : le caractère profondément social du rire et la possibilité de reconstruction qu'il offre. Il se construit néanmoins dans la conflictualité autant que dans l'échange puisque chacun sape les prétentions totalisantes, car investissant l'ensemble de l'univers symbolique, de l'autre. Au cœur de cette tension se trouve l'un des ressorts intimes de la mécanique de South Park, proposant à la fois un pur appel ludique et un véritable questionnement éthique, chacun restant libre d'en retirer ce qu'il désire. Si le jeu entre l'humour et la morale ouvre bien un espace particulier, 
c'est donc celui de l'épanouissement de la liberté subjective, position morale fondatrice s'il en est.

\section{Subversion et conservatisme}

Contrairement à ce que l'on pourrait penser au premier abord, la subversion ne consiste ni en l'anarchie, ni en une position nihiliste. Elle exige un ordre foncièrement sérieux ${ }^{1}$, un système de valeur bien hiérarchisé afin d'en permettre le renversement. La subversion se trouve donc être le stade ultime du positionnement moral. Elle y reste cependant totalement immergée. C'est en ce sens que se pose la question du conservatisme : en ne faisant que reprendre le canevas des valeurs traditionnelles pour le retourner, ne risque-t-on pas de le perpétuer sans véritablement en remettre en cause la légitimité ?

De fait, South Park nous offre d'excellents exemples de renversement des valeurs morales contemporaines, tels que la représentation ridicule de toutes les institutions sur lesquelles se fondent nos démocraties (presse, armée, associations en tout genre, partis politiques, enseignement...) ou un traitement humoristique de faits d'actualité graves (élection présidentielle, guerre en Irak, immigration...). Mais, dans le même temps, il termine la plupart de ses épisodes par des moralités célébrant le fait de continuer à vivre comme nous le faisons.

Aussi, il paraît évident que la transgression dans cette série animée est plus symbolique que morale. Jamais le meurtre, la paresse ou la drogue ne sont prônés comme bien, ni l'entraide, l'honnêteté ou la famille comme mal. Par contre, les entités ayant autorité se trouvent ridiculisées dans tous les cas de figure, qu'il s'agisse de figures religieuses, politiques, médiatiques... Si il y a subversion, elle ne se trouve donc pas dans les idées mais bien dans le traitement que l'on y fait subir à nos constructions mythologiques. Le diable souffre de solitude et est manipulé par Saddam Hussein, Jésus est un minable présentateur d'émission de télévision, les journalistes racontent n'importe quoi, les stars sont folles et dangereuses, les policiers sont totalement incompétents...

Cette constatation entraîne plusieurs réflexions : premièrement, encore une fois, South Park se positionne sur le terrain de la représentation et non pas de la rationalité ; deuxièmement, cette forme de subversion réactive le fond culturel commun aux téléspectateurs tout en

1 LiPOVETSKY, G., L'ère du vide. Essais sur l'individualisme contemporain, Paris, Gallimard (coll. «Folio / Essais »), 1983, p. 233. 
le bousculant ; troisièmement, un tel jeu n'est autorisé que parce qu'il est soutenu par un système de valeurs parfaitement actif, composé de relativisme et de liberté vis-à-vis des références culturelles. Par conséquent, d'un point de vue moral, la subversion dans ce dessin animé reste confinée à des objets secondaires. Nous n'avons pas affaire à un renversement de toutes les valeurs, mais de toutes les images, ce qui maintient l'ordre des valeurs en place tout en endossant le masque du changement. Sans doute ne peut-on exiger d'une fiction télévisuelle de faire la révolution. Cependant, peut-être peut-on s'interroger sur l'impact d'une telle représentation de la transgression sur notre capacité à imaginer d'autres sociétés.

Quoiqu'il en soit, le jeu entre la subversion des représentations et le maintien de l'ordre moral permet au dessin animé de rejeter les stéréotypes tout en maintenant une position de bon sens vis-à-vis des problèmes concrets. En effet, il opère avant tout une distinction nette entre les images, qui constituent notre imaginaire symbolique et peuvent être torturées à souhait, et le réel, qui constitue le seul objet méritant notre attention. Peu importe que toutes nos représentations soient bafouées car il ne s'agit pas de la vraie vie, au même titre d'ailleurs que les objets médiatiques qui les alimentent en permanence. Par contre, les vrais gens ordinaires, vivant une vie ordinaire au cour d'une petite ville ordinaire, ça c'est réel et ça mérite donc qu'on en préserve les valeurs fondamentales.

Une telle conception dichotomique constitue une base du fonctionnement global de South Park.

\section{Bon sens et pragmatisme}

Nous avions défini plus haut la morale de notre dessin animé comme une morale pragmatique du bon sens commun. Ces termes ont été utilisés dans leur sens usuel. Il n'est pas superflu de s'attarder un instant sur leur définition plus systématique afin d'en déceler les éventuels enjeux idéologiques et sociologiques. Cette partie admettra donc nos prémisses comme vraies ; même si cela ne signifie en aucun cas qu'il s'agisse de la seule qualification possible à la morale dans cette série. 
Selon l'encyclopédie philosophique universelle, le bon sens peut se définir comme la « faculté de juger de manière droite, sans faire appel à un raisonnement complexe, ni à des connaissances spécifiques ${ }^{1}{ }$.

En ce sens, il s'opposerait donc à la connaissance par érudition, ainsi qu'à la raison, en ce que « le bon sens, lui, accèderait à son objet de façon plus directe et intuitive et pourrait prétendre à l'assentiment du plus grand nombre, en particulier dans ce qui touche à la morale et aux jugements de valeur ${ }^{2} \gg$.

On ne peut manquer d'y voir la correspondance avec la conception de la morale que défend South Park tout au long de ses épisodes. Jamais la série ne prendra le parti de l'érudition ou d'une sagesse qui ne serait pas immédiatement accessible à tous. Mais toujours elle défendra une rationalité immédiate, tout en la présentant toutefois comme étant à redécouvrir sous l'amas d'idéologies qui en empêche l'expression simple et évidente.

Le sens commun, à présent, est défini comme « un ensemble de jugements communément (ou universellement) acceptés par les membres d'une communauté humaine (ou par l'humanité toute entière) ${ }^{3}$ ».

Il présuppose donc l'idée d'une communauté culturelle au sein de laquelle ces jugements seraient tacitement acceptés par tous sans qu'il ne soit nécessaire d'en faire état, sauf lorsqu'il s'agit d'user de leur autorité. Une telle idée peut sembler aller à l'encontre du caractère antiinstitutionnel et anti-idéologique de la série. Pourtant, nous avons suffisamment démontré que celle-ci nous proposait avant tout un univers de représentation destiné à célébrer le mode de vie qui en permet l'existence. Par conséquent, tout aussi contradictoire que cela puisse paraître, en prônant la primauté de l'individu sur toute forme de communauté, South Park célèbre les valeurs de la culture dont il est issu.

A partir de ces deux définitions, nous pouvons donc déjà remarquer deux caractéristiques à la morale véhiculée par ce dessin animé. Tout d'abord, nous avons affaire à une position anti-intellectualiste défendant l'immédiateté et l'accessibilité au plus grand nombre de l'intuition éthique. Ensuite, cette accessibilité directe est permise par le simple fait d'appartenir à la même culture. Ces deux points sont particulièrement

1 SOULEZ, P., Sens (bon -), in Encyclopédie philosophique universelle : les notions philosophiques, Tome 2, Paris, P.U.F., 1990, pp. 2347-2348.

2 Ibid, pp. 2347.

3 SCHULTHESS, D., Commun (sens -), in Encyclopédie philosophique universelle: les notions philosophiques, Tome 2, Paris, P.U.F., 1990, pp. 360-361. 
interpellants : premièrement parce qu'ils permettent que s'incarne une nouvelle forme d'ethnocentrisme, deuxièmement, parce que la remise en cause de celui-ci n'est plus permise à la raison, qui n'a plus droit de cité, et troisièmement parce que ces évidences culturelles sont déversées comme telles aux quatre coins du globe.

Pour terminer, penchons-nous sur le dernier terme dont nous avions usé pour définir la morale proposée par la série. Le terme «pragmatique » vient du grec pragma, qui signifie l'action, et se rapporte à une doctrine philosophique à part entière, le pragmatisme. Il s'agit d'ailleurs de la seule doctrine née au Etats-Unis. C. S. Peirce fut à l'origine de ce courant, en proposant avant tout une théorie des signes et de la vérité pour laquelle la conception d'un objet se limite à la conception de ses effets pratiques. Avec W. James, les aspects anti-intellectualistes et subjectivistes de cette doctrine s'accentua et, peu à peu, «le mot "pragmatisme" a finit par désigner dans la langue courante une attitude qui privilégie les questions pratiques au détriment des questions théoriques, ou qui réduit celles-ci à celles-là ${ }^{1} »$.

Par conséquent, «au sens courant, et par extension, le pragmatisme est une manière réaliste de penser et d'agir, méfiante à l'égard des idées générales, franchement hostile aux utopies ${ }^{2} »$.

Une telle ressemblance avec les observations que nous avons pu mener tout au long de l'approfondissement du point de vue moral proposé par South Park apparaît presque suspect. Pourtant, loin d'être un guet-apens, destiné à clore le débat, ces définitions doivent nous permettre de mieux comprendre les enjeux en présence. Ainsi, tout d'abord, nous pouvons remarquer que ces trois notions sont avant tout des termes du langage courant dont l'usage philosophique reste problématique. Notre dessin animé est loin d'avoir adopté les questions et les exigences de la philosophie morale. Il reste une pratique avant d'être un message. Rien ne permet donc de supposer que les auteurs connaissent et défendent une position éthique bien définie. Mais cela ne fait que démontrer l'impact que celle-ci a fini par provoquer au cœur des représentations de la communauté. Ensuite, l'origine de cette doctrine démontre à quel point un tel mouvement est, lui aussi, contingent. Il ne s'agit en rien d'une évolution naturelle de la pensée, mais bien d'une

1 Chauviré, C., Pragmatisme, in Encyclopédie philosophique universelle: les notions philosophiques, Tome 2, Paris, P.U.F., 1990, pp 2016-2017.

2 Dictionnaire de philosophie, GODIN, C., Poitiers, Fayard/Editions du temps, 2004, p. 1017. 
vision du monde culturellement déterminée et dont il importe de relativiser le caractère d'évidence ; même si une telle démarche ne peut avoir qu'un impact limité sur un tel modèle, rendu vrai par les pratiques dont il permet l'épanouissement.

A côté de ces prémisses, constituant la forme sous laquelle se développe chacun des messages de South Park, nous avons pu déterminer également le principe fondamental présidant à l'élaboration de ceux-ci. Il s'agit de la primauté de l'individu. Ce principe constituant l'objet récurrent d'une réflexion prétendant à l'évidence du bon sens démontre le caractère suspect d'un tel fondement. Comment expliquer, en effet, que cette faculté de juger sans faire appel à aucune idéologie en particulier en arrive à chaque fois à défendre la liberté individuelle ? Ce fait seul démontre le caractère problématique car culturellement déterminé de la morale de la série.

\section{Conclusion}

Nous avions commencé cet article par une brève délimitation de ce que l'on peut entendre par morale. Les différents éléments mis en lumière par notre étude de la série animée South Park s'inscrivent parfaitement dans ce domaine de préoccupations. Nous avons, en effet, démontré l'importance accordée aux entités fondatrices que sont le sujet, la raison et la société. Néanmoins, nous n'avons pas affaire à l'élaboration d'un système mais bien à un évènement, à la mise en scène de situations morales. Cette constatation, évidente, a peut être plus d'importance qu'elle n'y paraît. En effet, il semble que tout, dans la forme même du dessin animé, concourt à soutenir une position pragmatiste du bon sens commun.

La série télévisée ne favorise-t-elle pas par essence le quotidien, le particulier, le contexte ? Et si il s'agissait là du mode d'expression privilégié d'une culture pragmatiste ? Sans doute cela mériterait-il un examen plus approfondi. Nous nous contenterons donc pour l'instant d'en émettre l'hypothèse.

Trois caractéristiques renforcent cependant notre conviction. Tout d'abord, nous avons vu à quel point le sujet, vu comme individu libre, est fondamental pour l'horizon dans lequel s'insère South Park. Il est clairement la condition d'existence de la série et tout s'applique à le 
renforcer. Or, l'aspect individuel est particulièrement soutenu par la forme de la série télévisée, que ce soit par l'incarnation nécessaire dans des personnages ou par la manière dont elle se propose au public dans un mode de consommation personnalisé.

Ensuite, sur le plan de la raison, nous avons affaire à un système dichotomique entre le quotidien contextualisé des gens ordinaires, qui représente l'univers réel, et puis les abstractions politiques, idéologiques et médiatiques, institutions, systèmes de pensées et célébrités, qui ne sont que des images, des illusions dont il faut se défaire pour accéder au Vrai. En ce sens, nous nous trouvons face à une morale qui, quoique rejetant le fondement rationnel, logique et systématique, se présente exactement comme toutes les autres, c'est-à-dire comme seule capable de se défaire des illusions pour atteindre la vérité, vérité qui s'apparente ici à l'idée d'authenticité accolée au petit peuple. Ce rejet de toutes les idéologies et l'accent mis sur la capacité pour tous, sans connaissances préalables, et surtout pour les gens ordinaires, d'accéder à la vraie moralité s'adapte idéalement à la situation du dessin animé qui doit s'adresser au plus grand nombre et valoriser son public.

Enfin, d'un point de vue social, la série est clairement l'expression de sa culture. Et même si cette culture est minimale, comme support à la liberté individuelle, elle représente la raison d'être, autant que l'expression en tant que telle, de la série télévisée. Par conséquent, la subversion qu'elle propose ne peut en rien permettre l'ouverture à d'autres horizons. L'univers proposé reste parfaitement clos sur lui-même. Le problème peut dès lors se poser quant aux capacités d'imagination qu'il permet de développer. Là où des penseurs comme Richard Rorty ou Gianni Vattimo voient dans les productions culturelles, artistiques ou de sciences humaines, la possibilité pour l'individu de s'ouvrir à d'autres mondes, d'autres modes de vie, d'autres visions du monde ; il semble que South Park propose l'image un peu sulfureuse de la subversion tout en permettant de rester installé confortablement au sein de sa propre culture. Une telle ambiguïté ne doit évidement pas faire peur tant que le dessin animé s'insère dans une consommation culturelle diversifiée. Cependant, s'il se révélait être la seule référence contestataire, nous pourrions sans doute nous interroger sur les possibilités qui nous resteraient ouvertes d'envisager un autre monde.

La place qu'offre South Park aux trois piliers de la morale nous démontre donc, encore une fois, à quel point cette fiction s'insère dans 
une vision du monde pragmatiste fondée sur le bon sens commun. Et il nous est permis de nous interroger sur la congruence qui pourrait exister entre cet univers de pensée et le mode de représentation que propose la série télévisée.

\section{Bibliographie}

- Dictionnaire de philosophie, GODIN, C., Poitiers, Fayard/Editions du temps, 2004, p. 1017.

- BRUCKNER, P., La tentation de l'innocence, Paris, Grasset, 1995.

- CHAUVIRÉ, C., Pragmatisme, in Encyclopédie philosophique universelle: les notions philosophiques, Tome 2, Paris, P.U.F., 1990, pp 2015-2017.

- CRITCHLEY, S., De l'humour, (trad. N. PINET), Paris, Kimé, 2004.

- DuClos, D., Le complexe du loup garou : la fascination de la violence dans la culture américaine, Paris, La découverte, 1994.

- DUPONT, F., Homère et Dallas, Paris, Hachette, 1991.

- LIPOVETSKY, G., L'ère du vide. Essais sur l'individualisme contemporain, La Flèche, Ed. Gallimard (coll. « Folio / Essais »), 1983.

- Schoentjes, P., Poétique de l'ironie, Paris, Seuil (coll. « Points / Essais »), 2001.

- SCHULTHESS, D., Commun (sens -), in Encyclopédie philosophique universelle : les notions philosophiques, Tome 2, Paris, P.U.F., 1990, pp. 360-361.

- SOULEZ, P., Sens (bon -), in Encyclopédie philosophique universelle: les notions philosophiques, Tome 2, Paris, P.U.F., 1990, pp. 2347-2348. 
